\title{
Spatio-Temporal Model of Extreme Rainfall Data in the Province of South Sulawesi for a Flood Early Warning System
}

\begin{abstract}
In this study, we model extreme rainfall to study the high rainfall events in the province of South Sulawesi, Indonesia. We investigated the effect of the El Nino South Oscillation (ENSO), Indian Ocean Dipole Mode (IOD), and Madden-Julian Oscillation (MJO) on extreme rainfall events. We also assume that events in a location are affected by events in other nearby locations. Using rainfall data from the province of South Sulawesi, the results showed that extreme rainfall events are related to IOD and MJO.
\end{abstract}

Keywords: spatio-temporal model, extreme rainfall, province of South Sulawesi, El Nino South Oscillation, Indian Ocean Dipole Mode, and Madden-Julian Oscillation

Received: 18 September 2020; accepted: 19 November 2020

(C) 2021 Authors. This is an open access publication, which can be used, distributed and reproduced in any medium according to the Creative Commons CC-BY 4.0 License.

\footnotetext{
1 Hasanuddin University, Faculty of Technic, Department of Civil Engineering, Makassar, Indonesia, email: bambangbakri@gmail.com, ORCID ID: https://orcid.org/0000-0001-9006-2018

2 Hasanuddin University, Faculty of Mathematics and Natural Sciences, Department of Mathematics, Makassar, Indonesia, email: ririn.adam@gmail.com

3 Hasanuddin University, Department of Mathematics, Faculty of Mathematics and Natural Sciences, Makassar, Indonesia, email: amran@science.unhas.ac.id (corresponding author),

ORCID ID: https://orcid.org/0000-0002-9832-8738
} 


\section{Introduction}

Extreme rainfall has a detrimental effect on human life and the environment. Extreme rainfall is that which rarely occurs in a location within a certain time [1]. There are two approaches used in determining extreme rainfall, namely Peak Over Threshold (POT) and Block Maxima (BM) [2]. In the POT approach, extreme rainfall is expressed as rainfall that is greater than the threshold value. Meanwhile, in the BM approach, extreme rainfall is expressed as the maximum value in a block of time.

Extreme rainfall can be divided into high extreme rainfall and low extreme rainfall. High rainfall is associated with floods or landslides, while low rainfall is associated with drought. Both types of extreme rainfall have adverse effects on human life and the environment. High extreme rainfall is defined as rainfall that is more than or equal to the $75^{\text {th }}$ or $90^{\text {th }}$ percentile $[1,3]$.

Extreme rainfall in Indonesia is strongly linked to the phenomena of global climate change such as the El Nino South Oscillation (ENSO) [4], Indian Ocean Dipole Mode (IOD) [5], and Madden-Julian Oscillation (MJO) [6]. This is because Indonesia is one of the tropical regions between the Pacific Ocean and the Indian Ocean and the continents of Asia and Australia. This study aims to model the rate of extreme rainfall events related to the effects of climate change on 21 areas in the province of South Sulawesi in order to construct an early warning system.

The number of extreme rainfall events is a counting process that can be modeled into the Poisson model [7]. The Poisson processes with constant intensity are called Homogeneous Poisson processes, while Poisson processes with time-dependent intensities are called non-homogeneous Poisson processes (NHPP). The number of extreme rainfall events over time certainly varies because of climate change, in other words they are not constant. Thus, non-homogeneous Poisson processes characterized by time-dependent intensity functions are realistic enough to be applied when modeling extreme rainfall phenomena.

The NHPP model has been applied in various disciplines, for example model-

ing the arrival rate of containers in port operations and management [8], analyzing ozone behavior [9], analyzing rainfall occurrence [10], and modeling the frequency of extreme rainfall [11]. However, the studies that have been carried out are mostly focused on the development of time-dependent models, whilst extreme rainfall modeling involves data that is observed at different times and locations so that often observations in a location are affected by observations in other nearby locations. Thus, the addition of spatial effects to a model should be considered.

Some researchers add spatial effects to their research model to explain the possible correlations and sources of variance that are not explained in the model. Huang et al. [12] applied Conditional Auto-Regressive (CAR) to explain spatial correlation in modeling $\mathrm{N}_{2} \mathrm{O}$ emissions. Rusworth et al. [13] present a new model for estimating the effects of air pollution on human health by using the spatial 
effect of CAR on Spatio-temporal model. Sharkey and Winter [14] add the spatial effect of CAR distribution on the Generalized Pareto Distribution (GPD) parameter for precipitation modeling. Marco et al. [15] use CAR as a spatial effect in his research on modeling drug crime. The addition of the spatial effect of CAR on the models of these studies was constructed using the Bayesian hierarchy framework. A Bayesian hierarchy is considered a flexible framework and allows the incorporation of various sources of uncertainty [14].

\section{Data}

The data used in this study are daily rainfall data at 60 locations in 21 areas in the province of South Sulawesi obtained from the Global Satellite Mapping of Precipitation or GSMaP (ftp://hokusai.eorc.jaxa.jp). This research analyses extreme rainfall events for 8 years from 2009 to 2016 . Three important climatic factors: the El Nino Southern Oscillation (ENSO), the Indian Ocean Dipole (IOD), and the MaddenJulian Oscillation (MJO) are considered in this study to understand their effects on extreme rainfall occurrence. ENSO and IOD data were obtained from the National Oceanic and Atmospheric (NOAA) and MJO data from the Bureau of Meteorology. Figure 1 shows the districts in the province of South Sulawesi, Indonesia and rainfall station locations.

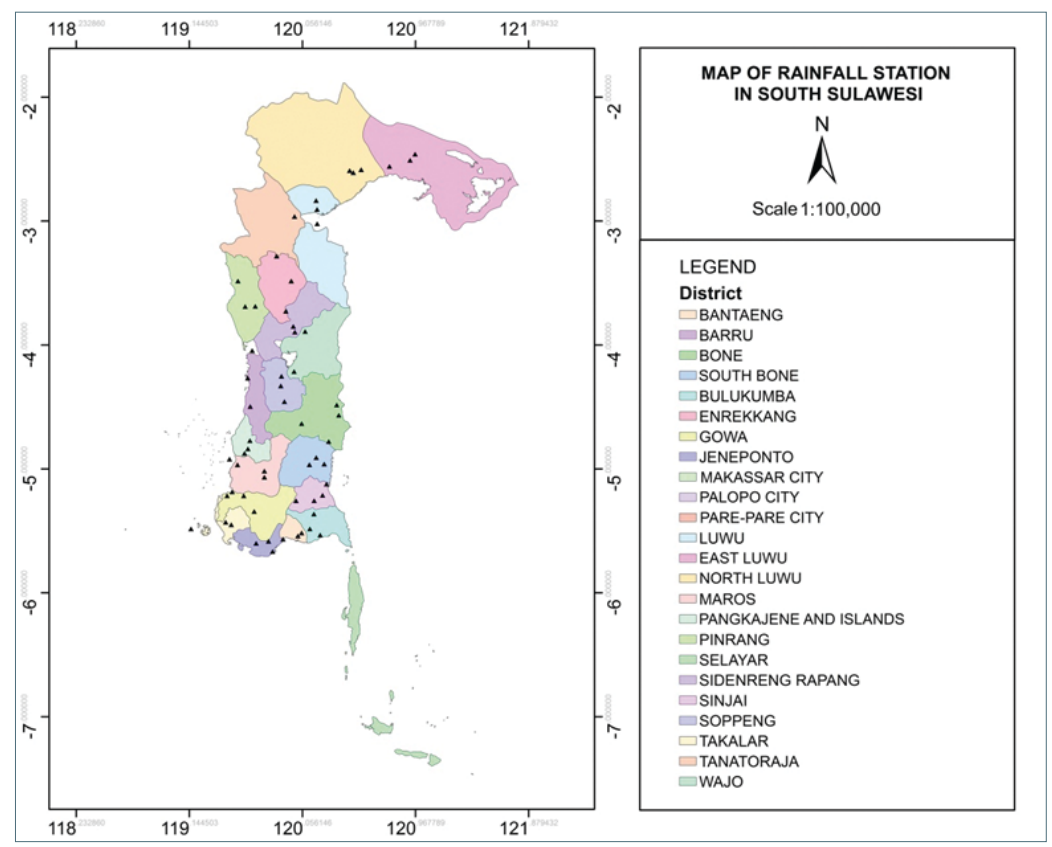

Fig. 1. Map of rainfall station locations in the province of South Sulawesi 


\section{Methodology}

The steps in this study are as follows: Collecting data, determine extreme rainfall data by calculating the $75^{\text {th }}$ percentile value in a day's rainfall, counting the number of days with rainfall more than or equal to the $75^{\text {th }}$ percentile (D75) every month, identify spatial correlations with Moran's I test, construct NHPP models for the number of extreme rainfall events D75 with three independent variables with the addition of spatial effects of CAR in the Bayesian Hierarchy framework, parameter estimation using WinBugs Version 3.0.2 software, and interpretation of research results.

\section{Extreme Rainfall Modeling}

Modeling the rate of extreme rainfall events is carried out within the Hierarchical Bayesian framework. Non-homogeneous Poisson modeling with spatial effects using the Bayesian hierarchy method consists of three steps, namely the data modeling step, the process modeling step, and the prior distribution selection step. The hierarchical modeling structure is described as follows:

Let $y_{t i}$ denotes the number of extreme rainfall day in time $t, t=1,2, \ldots, T$ and area $i$, $i=1,2, \ldots, n . y_{t i}$ is Poisson distribution data, which can be shown through the Kolmogorov-Smirnov test. $y_{t i}$ is modeled as Poisson distribution data with the parameter $\lambda_{t i}$, then the data modeling step can be written as follows:

$$
y_{t i} \sim \operatorname{Poisson}\left(\lambda_{t i}\right),
$$

where $\lambda_{t i}$ depends on the time $t$ and the location $i$ of the event with the likelihood function as follows:

$$
L(\theta)=f(Y \mid \lambda)=\prod_{t=1}^{T} \prod_{i=1}^{n} \frac{\lambda_{t i}^{y_{i t}} e^{-\lambda_{t i}}}{y_{t i} !}
$$

In process modeling, it is assumed that the rate of extreme rainfall events $\lambda_{t i}$ is based on spatial processes since neighboring locations have more similar characteristics than locations farther away, so that $u_{i}$ as spatial random effects are added to the model. Also, three climate covariates that are thought to affect extreme rainfall events: MJO, IOD, and ENSO are also included in the model thus the model can be written as follows:

$$
\log \lambda_{t i}=\beta_{0}+\beta_{1} M J O_{t i}+\beta_{2} N I N O_{t i}+\beta_{3} I O D_{t i}+u_{i},
$$

where $\beta_{0}$ is global intercept, $\beta_{1^{\prime}} \beta_{2^{\prime}} \beta_{3}$ are regression coefficients, MJO $_{t i^{\prime}}$ NINO $_{t i^{\prime}}$ $I O D_{t i}$ are predictors at time $t$ in location $i$ and $u_{i}$ are spatial random components at location $i$ modeled with priors conditional autoregressive (CAR) distribution. 
The use of a log-link in parameter estimation aims to ensure the parameter value is a non-negative number. Banerjee et al. [16] in Sharkey and Winter [14] write the CAR model with conditional prior for $u_{i}$ as:

$$
u_{i} \mid u_{-i} \sim N\left(\sum_{j \in n} \frac{u_{-i}}{w_{i+}}, \frac{\tau^{2}}{w_{i+}}\right) \text {, }
$$

where $u_{i}$ is spatial random effect, $\tau^{2}$ is the variance, $w_{i+}=\sum_{j=1}^{n} w_{i j}$ with $w_{i+}$ is the number of neighbors of cell location $i, w_{i j}$ is spatial weight on the spatial weight matrix $W$. A real $n \times n$ matrix defining spatial proximity between cells $i$ and $j$ with $w_{i j}=1$ if location $i$ and $j$ are adjacent and $w_{i j}=0$ otherwise.

The last step in hierarchical modeling is defining priors and assumed that the parameters at each step of the model are independent. Assuming there is no prior knowledge of one of the parameters in the model, it is chosen to set non-informative prior for $\beta_{k}=\left(\beta_{0^{\prime}}, \beta_{1^{\prime}}, \beta_{2^{\prime}} \beta_{3}\right)$ with consideration of $\beta_{k} \sim N(\mu, \varphi)$ where hyperprior $\mu$ and $\varphi$ are normally distributed with mean-centered on zero and a large and fixed variant [14]. The definition of the prior is described as follows: Parameter $\beta_{k}=\left\{\beta_{0^{\prime}} \beta_{1^{\prime}} \beta_{2^{\prime}} \beta_{3}\right\}$ are priors with Normal distribution, $\beta_{k} \sim N(\mu, \varphi), \mu \sim N(0,100)$, $\varphi \sim N(0,100), \tau \sim \operatorname{Gamma}(0.5,0.0005)$.

Proportionally, the posterior distribution is the product of the likelihood function with prior. The parameters that will be estimated are written as:

$$
\theta=\left\{\beta_{0}, \beta_{1}, \beta_{2}, \beta_{3}, u_{i}\right\}
$$

with hyperparameter:

$$
\psi=\{\mu, \varphi, \tau\}
$$

thus the likelihood function in this model is:

$$
f\left(y \mid \beta_{0}, \beta_{1}, \beta_{2}, \beta_{3}, u_{i}, \mu, \varphi, \tau\right)=\prod_{t=1}^{T} \prod_{i=1}^{n} f\left(y_{t i} \mid \beta_{0}, \beta_{1}, \beta_{2}, \beta_{3}, u_{i}, \mu, \varphi, \tau\right) .
$$

Therefore, the posterior distribution can be written as follows.

$$
\begin{gathered}
f(\boldsymbol{\theta}, \boldsymbol{\psi} \mid \boldsymbol{y})=f(\boldsymbol{y} \mid \boldsymbol{\theta}, \boldsymbol{\psi}) \times f(\boldsymbol{\theta} \mid \boldsymbol{\psi}) \times f(\boldsymbol{\psi}), \\
f(\boldsymbol{\theta}, \boldsymbol{\psi} \mid \boldsymbol{y})=f\left(y_{t i} \mid \beta_{0}, \beta_{1}, \beta_{2}, \beta_{3}, u_{i}, \mu, \varphi, \tau\right) \times f\left(\beta_{0} \mid \mu, \varphi\right) \times f\left(\beta_{1} \mid \mu, \varphi\right) \times \\
\times f\left(\beta_{2} \mid \mu, \varphi\right) \times f\left(\beta_{3} \mid \mu, \varphi\right) \times f\left(u_{i} \mid \tau\right) \times f(\varphi) \times f(\tau) .
\end{gathered}
$$

Model parameters are estimated by means of the Bayes method using the Markov Chain Monte Carlo (MCMC) algorithm with WinBugs version 3.0.2 software. 


\section{Result}

The parameter estimation results with the Bayesian method are good when the estimated parameters converge. The convergence of the estimation results was observed by the visual inspection of trace samples for each chain, density, history, autocorrelation, and convergence statistics of Gelman-Rubin. The estimation results for the high extreme rainfall rate models are obtained by carrying out an MCMC run of 10.000 iterations with 1.000 burn-in period. Trace sample plots for each chain, density, history, autocorrelation, and convergence statistics of Gelman-Rubin show that MCMC is mixing well.

The estimation results for the number of extreme rainfall days (D75) model are presented in Table 1. The first column in each Table is the covariate parameter (factors) that are thought to affect extreme rainfall events rate, the mean column shows the magnitude of the model parameter value, and for the next three columns, namely val2.5\% (credible lower limit interval), median, and val97.5\% (upper limit of the credible interval) is the estimated value on the $95 \%$ credible interval. Covariates with values at credible intervals that do not contain zero value are considered to significantly affect the rate of the number of extreme rainfall days.

Table 1. Parameter posterior estimation results for the rate of D75 model

\begin{tabular}{||c|c|c|c|c|c||}
\hline Parameter & Mean & $\begin{array}{c}\text { Standard } \\
\text { deviation }\end{array}$ & Val2.5\% & Median & Val97.5\% \\
\hline \hline$\beta_{0}$ & 0.6365 & 0.0563 & 0.5274 & 0.6368 & 0.7469 \\
\hline$\beta_{1}$ & 0.1864 & 0.0566 & 0.0749 & 0.1863 & 0.2972 \\
\hline$\beta_{2}$ & -0.0005 & 0.0181 & -0.0367 & -0.0007 & 0.0349 \\
\hline$\beta_{3}$ & -0.7952 & 0.0645 & -0.9203 & -0.7945 & -0.6697 \\
\hline$\tau$ & 11.9300 & 5.5690 & 4.5500 & 10.7900 & 25.7800 \\
\hline
\end{tabular}

Based on the estimation results in Table 1, what is stated to affect the rate of D75 in South Sulawesi with a confidence interval of $95 \%$ is MJO and IOD. This can be seen from the results of estimating the parameters $\beta_{1}$ and $\beta_{3}$ whose values do not contain zero on the credible interval. In contrast to ENSO, since it contains zero on the credible interval on the results of the $\beta_{2}$ parameter estpmation, it stated that ENSO did not show a significant result for D75 in the province of South Sulawesi for a period of 2009 to 2016. Meanwhile, the CAR parameter $(\tau)$ shows that there is a spatial dependency between neighboring locations of events.

The rate of the number of extreme rainfall days in the province of South Sulawesi can be modeled as follows:

$$
\log \lambda_{t i}^{D 75}=0.6365+0.1864 M J O_{t i}-0.7952 I^{2 O D} D_{t i}
$$


From the estimation results for D75 rates, it stated that for D75 behavior towards the climate covariate. The MJO and the IOD significantly influence while ENSO does not affect significantly. The CAR parameter $(\tau)$ significantly affects both the MJO and the IOD. It means that in the case of extreme rainfall events there are spatial dependencies in neighboring locations.

The description of D75 in the province of South Sulawesi (see Fig. 2) shows a pattern that varies over time. The presence of spatial dependencies occurred in the province of South Sulawesi. Several locations have similar colors.

a)

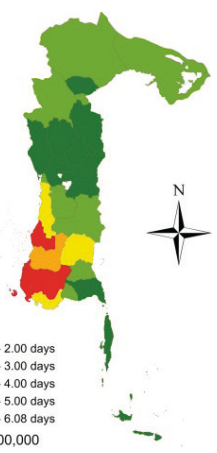

e)

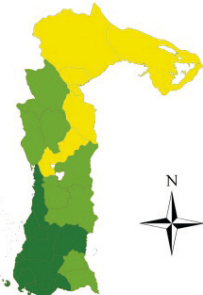

i)

Scale 1:100,000

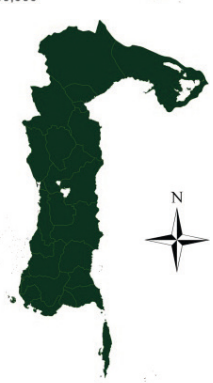

Legend

mean $0.13-0.50$ day Scale $1: 100,000$ b)

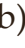
)

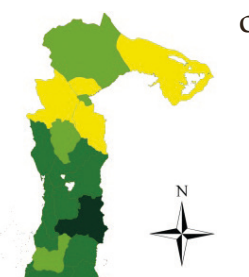

c)

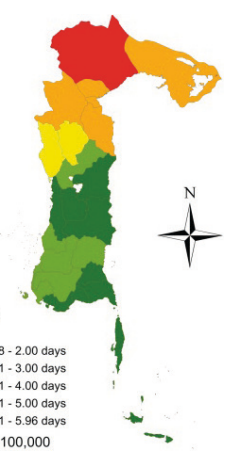

f)
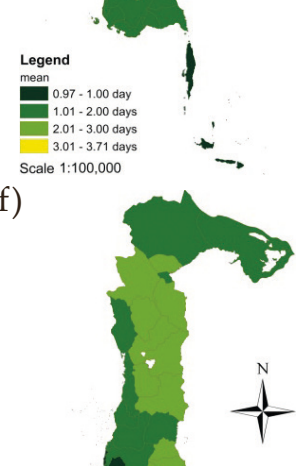

g)
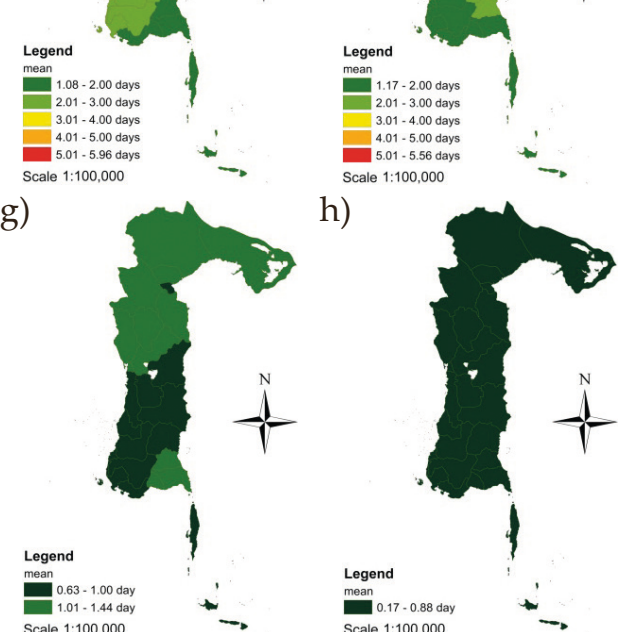

k)

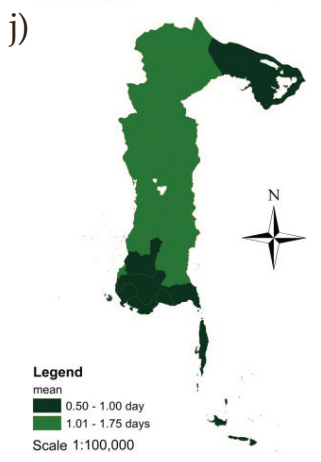

d)

h)
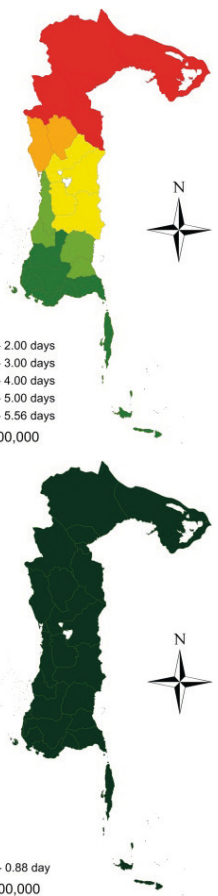

1)

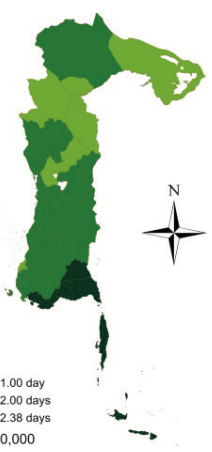

)

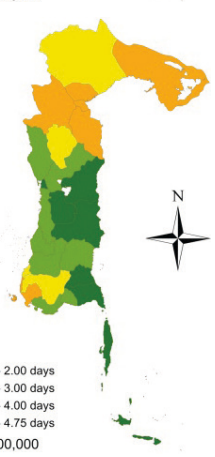

Fig. 2. Map of the high extreme rainfall rate in the period 2009 to 2016:

a) January, b) February, c) March, d) April, e) May, f) June, g) July,

h) August, i) September, j) October, k) November, l) December 
The rate of D75 in the province of South Sulawesi in the period 2009 to 2016 ranges from one to six days (see Fig. 2). The maximum rate of extreme rainfall days in six days and this occurs in January and April, with the rate of D75 in one-day for August and September. The variation in the rate of occurrence is certainly related to the geography and topography at the scene. Although globally the rate of extreme rainfall events is significantly affected by MJO and IOD, extreme rainfall events in several locations in South Sulawesi are influenced by the local nature of the location.

\section{Discussion}

The modeling of extreme rainfall events rate in this study was carried out by considering three climate covariates that were thought to affect extreme rainfall events: ENSO [4], IOD [5], and MJO [6]. Climate change that changes from time to time causes the incidence of extreme rainfall is not constant or changes over time. In that case, the NHPP is quite realistic to be used to model the rate of D75 that depend on changes in time.

Globally, 21 areas of observation in the province of South Sulawesi have spatial correlations with the location of their neighbors. This can be seen from the Moran index value $I=0.2990$. Thus the addition of spatial effects to rainfall modeling was carried out to explain the spatial random effects as carried out by [14]. Spatial dependence is done by selecting prior CAR on spatial random effects. In this study, prior CAR in the NHPP model is used to model high extreme rainfall events in the province of South Sulawesi.

The effect of the global climate on the rate of the number of extreme rainfall days is shown in Table 1. Both MJO and IOD showed a significant effect on the number of extreme rainfall days in the province of South Sulawesi. This can be seen from the results of estimating the parameters $\beta_{1}$ and $\beta_{3}$ whose values do not contain zero on the credible interval. This is in contrast to ENSO, because it contains zero on the credible interval on the results of the $\beta_{2}$ parameter estimation. ENSO has no significant effect on the rate of D75 in the province of South Sulawesi for the period of 2009 to 2016.

In general, ENSO has a varied influence on rainfall intensity and the number of rainy days both spatially and temporally. According to Deni et al. [17], ENSO has a major effect on rainfall anomalies in Indonesia. In the east of Indonesia, ENSO was correlated with rainfall in that location [18]. This study shows that the rate of D75 in the province of South Sulawesi for a period of 2009 to 2016 is only significantly influenced by MJO and IOD, while ENSO does not have a significant effect. It can happen when ENSO merges concurrently with MJO or IOD or even both. Jones et al. [19] stated that MJO in the active phase led to increased extreme rainfall, where MJO generally tends to be most active during the ENSO neutral phase and experiences 
a resting phase when ENSO strengthens. Also, IOD reduces the impact of ENSO, while merging at the same time [20]. MJO is stronger during negative IOD compared with positive IOD [21]. When IOD is negative, MJO increases the probability of the occurrence of high extreme rainfall. When IOD is positive, the modulation of the wet days by MJO becomes weaker.

\section{Conclusion}

This study shows that for the period of 2009 to 2016, IOD and MJO are associated with the rate of the number of extreme rainfall days. When there is a negative IOD, the incidence of the number of extreme rainfall days tends to increase. $\mathrm{MJO}$ in the active phase affects the rate of high extreme rainfall events at the location it passes. The ENSO does not have a significant effect on the rate of D75 events. The random effect of spatial shows a significant effect, which means that the rate of the number of extreme rainfall days in the area of the province of South Sulawesi affects the rate of occurrence in neighboring locations.

\section{Acknowledgement}

We thank our anonymous reviewers for their valuable advice which has improved the quality of this paper. The authors acknowledge the Ministry of Research and Technology/National Agency for Research and Innovation (RISTEK-BRIN), Indonesia for funding this research via Grant PDUPT Hasanuddin University in 2020 with grant number 1516/UN4.22/PT.01.03/2020. We would like to gratefully acknowledge Global Satellite Mapping of Precipitation, for providing the data set used in this study.

\section{References}

[1] The Intergovernmental Panel on Climate Change (IPCC): Managing the Risk of Extreme Events and Disasters to Advance Climate Change Adaptation. Cambridge University Press, Cambridge, UK and New York, USA, 2012.

[2] Coles S.: An Introduction to Statistical Modeling of Extreme Values. Springer, Bristol 2001.

[3] Zhang Q., Li J., Singh V.P.: Application of Archimedean copulas in the analysis of the precipitation extremes: effects of precipitation changes. Theoretical and Applied Climatology, vol. 107, 2011, pp. 255-264.

[4] Hendon H.H.: Indonesian Rainfall Variability: Impacts of ENSO and Local AirSea Interaction. Journal of Climate, vol. 16, issue 11, 2003, pp. 1775-1790.

[5] Nur'utami M.N., Hidayat R.: Influences of IOD and ENSO to Indonesian Rainfall Variability: Role of Atmosphere-ocean Interaction in the Indo-pacific Sector. Procedia Environmental Sciences, vol. 33, 2016, pp. 196-203. 
[6] Hidayat R.: Modulation of Indonesian Rainfall Variability by the Madden-Julian Oscillation. Procedia Environmental Sciences, vol. 33, 2016, pp. 167-177.

[7] Keim B.D., Cruise F.J.: A Technique to Measure Trends in the Frequency of Discrete Random Events. Journal of Climate, vol. 11, 1998, pp. 848-855.

[8] Wong H.L., Hsieh S.H., Tu Y.H.: Application of Non-Homogeneous Poisson Process Modeling to Containership Arrival Rate. [in:] 2009 Fourth International Conference on Innovative Computing, Information and Control: ICICIC 2009; Kaohsiung, Taiwan, 7-9 December 2009, Institute of Electrical and Electronics Engineers, 2009, pp. 849-854.

[9] Achcar J.A., Rodrigues E.R., Paulino C.D., Soares P.: Non-homogeneous Poisson models with a change-point: an application to ozone peaks in Mexico city. Environmental and Ecological Statistics, vol. 17, 2010, pp. 521-541.

[10] Sirangelo B., Ferrari E., de Luca D.: Occurrence analysis of daily rain-falls through non-homogeneous poissonian processes. Natural Hazards and Earth System Sciences, vol. 11, 2011, pp. 1657-1668.

[11] Mondal A., Mujumdar P.P.: Modeling non-stationarity in intensity, duration and frequency of extreme rainfall over India. Journal of Hydrology, vol. 521, 2015, pp. 217-231.

[12] Huang X., Grace P., Hu W., Rowlings D., Mengersen K.: Spatial prediction of $\mathrm{N}_{2} \mathrm{O}$ emissions in pasture: a Bayesian model averaging analysis. PLoS One, vol. 8(6), 2013, e65039.

[13] Rusworth A., Lee D., Mitchell R.: A spatio-temporal model for estimating the long-term effects of air pollution on respiratory hospital admissions in Greater London. Spatial and Spatio-temporal Epidemiology, vol. 10, 2014, pp. 29-38.

[14] Sharkey P., Winter H.C.: A Bayesian spatial hierarchical model for extreme precipitation in Great Britain. 2017, arXiv:1710.02091v1.

[15] Marco M., Gracia E., López-Quílez A.: Linking Neighborhood Characteristics and Drug-Related Police Interventions: A Bayesian Spatial Analysis. ISPRS International Journal of Geo-Information, vol. 6(3), 2017, 65. https://doi. org/10.3390/ijgi6030065.

[16] Banerjee S., Carlin B., Gelfand A.: Hierarchical Modeling and Analysis for Spatial Data. Monographs on Statistics and Applied Probability, 101, Chapman and Hall/CRC Press, New York 2004.

[17] Deni O.L., Edy S., Sabaruddin S., Iskhaq I.: Respective Influences of Indian Ocean Dipole and El Niño-Southern Oscillation on Indonesian Precipitation. Journal of Mathematical and Fundamental Sciences, vol. 50, no. 3, 2018, pp. 257-272.

[18] Lee H.S.: General Rainfall Patterns in Indonesia and the Potential Impacts of Local Seas on Rainfall Intensity. Water, vol. 7(4), 2015, pp. 1751-1768.

[19] Jones Ch., Waliser D.E., Lau K.M., Stern W.: Global occurrences of extreme precipitation and the Madden-Julian Oscillation: observations and predictability. Journal of Climate, vol. 17(23), 2004, pp. 4575-4589. 
[20] Ashok K., Guan Z., Saji N.H., Yamagata T.: Individual and Combined Influences of ENSO and the Indian Ocean Dipole on the Indian Summer Monsoon. Journal of Climate, vol. 17, 2004, pp. 3141-3155.

[21] Pourasghar F., Oliver E.C.J., Holbrook N.J.: Modulation of wet-season rainfall over Iran by the Madden-Julian Oscillation, Indian Ocean Dipole and El NiñoSouthern Oscillation. International Journal of Climatology, vol. 39, 2019, pp. 1-12. https://doi.org/10.1002/joc.6057. 